\title{
Double autophagy modulators reduce 2-deoxyglucose uptake in sarcoma patients
}

\author{
Mau-Shin Chi ${ }^{1,3}$, Cheng-Yen Lee ${ }^{1}$, Su-Chen Huang ${ }^{1}$, Kai-Lin Yang ${ }^{1}$, Hui-Ling Ko ${ }^{1}$, \\ Yen-Kung Chen ${ }^{2}$, Chen-Han Chung ${ }^{3}$, Kuang-Wen Liao ${ }^{3}$, Kwan-Hwa Chi ${ }^{1,4}$ \\ ${ }^{1}$ Department of Radiation Therapy and Oncology, Shin Kong Wu Ho-Su Memorial Hospital, Taipei, Taiwan \\ 2 Department of Nuclear Medicine and PET Center, Shin Kong Wu Ho-Su Memorial Hospital, Taipei, Taiwan \\ ${ }^{3}$ Institue of Molecular Medicine and Bioengineering, National Chiao-Tung University, Hsinchu, Taiwan \\ ${ }^{4}$ School of Medicine and Institute of Biomedical Imaging and Radiological Sciences, National Yang-Ming University, \\ Taipei, Taiwan
}

Correspondence to:

Kwan-Hwa Chi, e-mail: M006565@ms.skh.org.tw

Keywords: hydroxychloroquine, sirolimus, soft tissue sarcoma

Received: April 21, $2015 \quad$ Accepted: August 26, 2015

Published: September 07, 2015

\section{ABSTRACT}

Rationale: According to the metabolic symbiosis model, cancer stromal fibroblasts could be hijacked by surrounding cancer cells into a state of autophagy with aerobic glycolysis to help provide recycled nutrients. The purpose of this study was to investigate whether combined treatment with the autophagy inhibitor: hydroxychloroquine (HCQ) and the autophagy inducer: sirolimus (rapamycin, Rapa) would reduce glucose utilization in sarcoma patients.

Methods: Ten sarcoma patients who failed first-line treatment were enrolled in this study. They were treated with $1 \mathrm{mg}$ of Rapa and $200 \mathrm{mg}$ of HCQ twice daily for two weeks. The standardized uptake values (SUV) from pretreatment and posttreatment [18F]-fluorodeoxyglucose positron emission tomography (FDG PET) scans were reviewed, and changes from the baseline SUVmax were evaluated.

Results: Based on FDG PET response criteria, six patients had a partial response; three had stable disease, and one had progressive disease. Nevertheless, none of them showed a reduction in tumor volume. The mean SUVmax reduction in the 34 lesions evaluated was $-19.6 \%(95 \% \mathrm{CI}=-30.1 \%$ to $-9.1 \%)$, while the mean volume change was $+16.4 \%(95 \% \mathrm{CI}=+5.8 \%$ to $+27 \%)$. Only grade 1 toxicities were observed. Elevated serum levels of lactate dehydrogenase were detected after treatment in most metabolic responders.

Conclusions: The results of reduced SUVmax without tumor volume reduction after two weeks of Rapa and HCQ treatment may indicate that non-proliferative glycolysis occurred mainly in the cancer associated fibroblast compartment, and decreased glycolytic activity was evident from Rapa + HCQ double autophagy modulator treatment.

\section{INTRODUCTION}

The reported worldwide incidence rate of soft tissue sarcoma ranges from 1.8 to 5 per 100,000 people per year [1]. Multimodality treatment is often provided to patients with primary disease, whereas chemotherapy is given to those with metastatic disease. Unfortunately, sarcoma is usually chemo-insensitive. In addition to the intrinsic chemo-insensitivity of sarcoma cells, the interaction between a tumor and its microenvironment, which triggers chemo-resistance, might be of importance [2]. Cancerassociated fibroblasts (CAFs), which constitute a substantial volume in a tumor microenvironment, are closely associated with invasiveness, drug resistance, and metastasis to fibroblasts, endothelial cells, or adipose tissue [3, 4].

Martinez-Outschoorn et al. proposed the autophagic tumor stroma model and reported that cancer cells could be seen as "metabolic parasites" for using oxidative stress 
to extract recycled nutrients from adjacent stromal cells $[5,6]$. CAFs could produce high-energy nutrients (e.g., lactate and ketones) that fuel mitochondrial biogenesis and oxidative metabolism in cancer cells. This novel energy transfer mechanism is termed the "reverse Warburg effect" [7,8]. Autophagy in the tumor stroma serves as a "battery" to fuel tumor growth, progression, and metastasis, often independent of angiogenesis [5]. Hydroxychloroquine (HCQ) or other autophagy/ lysosome inhibitors may be useful to therapeutically restore the "normal" CAFs by blocking their state of hyper-autophagy. Furthermore, induction of sarcoma cell autophagy with sirolimus (rapamycin, Rapa) could prevent them from using recycled nutrients [5]. Thus, concurrent administration of the two above-mentioned agents might block the metabolic parasite relationship within a sarcoma tumor microenvironment $[5,8]$. Such a strategy may effectively "starve" cancer cells, rendering them more vulnerable to cytotoxic therapy. The metabolic modulation and change in bioenergetic homeostasis of the tumor microenvironment could affect mitochondrial electron transport complexes and glycolysis-related pathways in cancer cells, thus altering reactive oxygen species and adenosine triphosphate synthesis, and decreasing their survival capability under chemotherapy stress $[9,10]$.

Since CAFs usually have the largest increase in glucose uptake, [18F]-fluorodeoxyglucose positron emission tomography (FDG PET) of tumors might specifically detect the tumor stroma rather than the tumor cells [11]. The purpose of this study was to demonstrate that a decrease in glucose metabolism could mirror the attenuating metabolic-parasite relationship between CAFs and sarcoma cells by Rapa and HCQ treatment.

\section{RESULTS}

The primary end point of this study was the SUVmax change after two weeks of Rapa and HCQ treatment. The secondary end point was the response rate after eight weeks of treatment. However, most patients discontinued treatment before response assessment because of progression of disease and slow recruitment of cases (13 patients from August 2012 to June 2014), so the study was closed prematurely. Ten patients were evaluable by sequential [18F]-FDG PET images at baseline and after two weeks of treatment. Patient characteristics of these ten patients are shown in Table 1. All of the patients tolerated Rapa and HCQ treatment well with only mild grade 1 adverse events and were observed after a median of two weeks treatment (range two to four weeks) (Table 2).

There were 34 lesions evaluable for changes of SUVmax; these changes are presented in Figure 1. The mean baseline SUVmax was 13.5 (95\% confidence interval $[\mathrm{CI}]=10.1-16.9)$, and the mean posttreatment value was $9.9(95 \% \mathrm{CI}=7.5-12.3 ; p<0.003)$. The mean reduction in SUVmax was $-19.6 \%(95 \% \mathrm{CI}=-30.1$ to $-9.1 \%$ ), and the median was $-23.7 \%$ (interquartile range, $-41.8 \%$ to $4.1 \%$ ). The changes of SUVmax versus volume changes form the ten enrolled patients are shown in Figure 2. The mean change of SUVmax was $-31.3 \%(95 \%$ $\mathrm{CI}=-51.9 \%$ to $-10.6 \%$ ) while the mean volume change on the same lesion was $+16.4 \%(95 \% \mathrm{CI}=+5.8 \%$ to + $27.0 \%$ ). The disparity between glucose consumption rate and proliferation need in the tumors suggested a metabolic reprogramming process might have occurred. According to the EORTC 1999 criteria, six patients had a partial response, three had a stable disease, and one had a progressive disease. The biochemical profile changes before and after two weeks of Rapa and HCQ treatment are shown in Figure 3. Overall, there were no significant changes in the fasting plasma glucose, total cholesterol, or triglyceride levels. However, as shown in Figure 3B, the lactate dehydrogenase (LDH) level was significantly increased after treatment. Interestingly, most patients with elevated LDH levels were metabolic responders (Figure 4).

\section{DISCUSSION}

This study demonstrated for the first time that a metabolic symbiosis relationship between CAFs and sarcoma cells might be altered by Rapa and HCQ treatment. An inhibition of glycolysis within the tumors without tumor growth was noted.

According to the metabolic symbiosis theory, cancer cells induce oxidative stress in adjacent fibroblasts, resulting in the onset of a myofibroblastic pro-autophagic phenotype [13]. This pro-autophagic phenotype in fibroblasts leads to a loss of mitochondria via autophagy, forcing CAFs to undergo aerobic glycolysis and provide lactate as energy source for cancer cells. Autophagy in the tumor stroma is also used by adjacent epithelial cells to fuel tumor growth via oxidative mitochondrial metabolism $[14,15]$. The autophagy inhibitor chloroquine (or HCQ) blocks the process in CAFs and stops the energy flow to sarcoma cells, while the autophagy inducer, Rapa, inhibits mammalian target of rapamycin (mTOR) signaling and induces autophagy in cancer cells to reduce tumor growth rate. The so-called autophagy paradox by concurrent administration of an autophagy inducer and inhibitor could be explained by the two-compartment model in which the two drugs were suggested to have different target preferences [16]. However, Rapa and HCQ combination may have a synergistic effect on the same target. As shown in Figure 5, the inhibition of the mTOR signal by RAPA decreased hypoxia-inducible factor 1-alpha (HIF- $\alpha$ ) and glucose uptake and therefore decreased glycolysis. The decreased glycolysis activated more autophagy in glycolysis-dependent cells and rendered them more vulnerable to HCQ treatment by accumulation of wasted products in lysosomes. The decreased glycolytic activity without a concomitant decreased proliferation rate suggested that the Rapa and HCQ combination might 
Table 1: Patient characteristics $(n=10)$

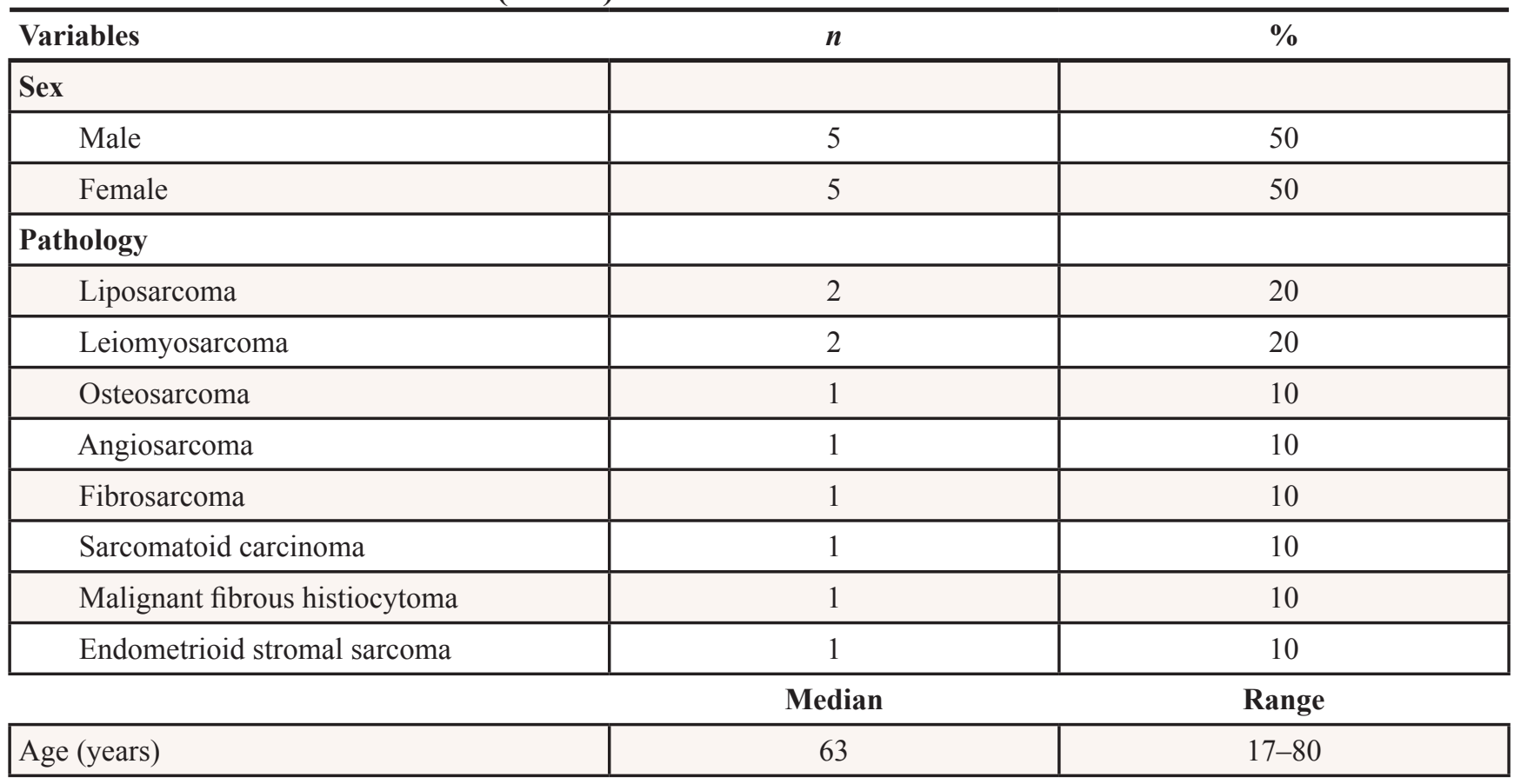

Table 2: Toxicities $(n=10)$

\begin{tabular}{|l|c|c|}
\hline Adverse effect & Grade 1 (n) & 0 \\
\hline Mucositis & 0 & 20 \\
\hline Skin rash & 2 & 0 \\
\hline Hair loss & 0 & 30 \\
\hline Nausea & 3 & 10 \\
\hline Diarrhea & 1 & 10 \\
\hline Constipation & 1 & 0 \\
\hline Dyspnea & 0 & 0 \\
\hline Somnolence & 0 & 0 \\
\hline Asthenia & 0 & 0 \\
\hline Fever & 0 & \\
\hline
\end{tabular}

not be able to block the proliferative signals in sarcoma cells. Figure 6 shows the coexistence of oxidative phosphorylation (OXPHOS) sarcoma cells and glycolytic sarcoma cells, similar to the metabolic coupling model proposed from osteosarcoma [17, 18]. Although the Rapa and HCQ combination decreased the glycolytic activity, the decreased lactate supply may have compensated by stimulating the OXPHOS function in the mitochondriarich sarcoma cells, based on the observed tumor proliferation and decreased glycolytic activity.

The inhibition of glucose metabolism assessed by [18F]-FDG PET without a decrease in tumor proliferation after Rapa plus HCQ treatment in sarcoma patients is somewhat surprising, because reduction of both SUVmax and SUV (mean) are generally regarded as indicative of decreased tumor viability. The observed decrease in glucose consumption on tumor area may have been largely due to the tumor microenvironment and therefore independent of tumor viability. This finding was novel in certain aspects. Firstly, this is the first report on the effect of combined Rapa and HCQ on glucose metabolism in cancer patients. To our knowledge, no publication is available regarding the effect of combined double autophagy modulators on glucose metabolism measured by $[18 \mathrm{~F}]-F D G$ PET in cancer patients. A Rapa analogue, everolimus, has been reported to decrease glucose uptake in few type of cancers $[19,20]$. The decrease in glucose uptake by everolimus has not been linked to the inhibition of glycolysis rather than cytotoxicity in any study. Secondly, our results provide functional image evidence of the metabolic parasite relationship proposed by Lisanti's group, namely that catabolic fibroblasts 


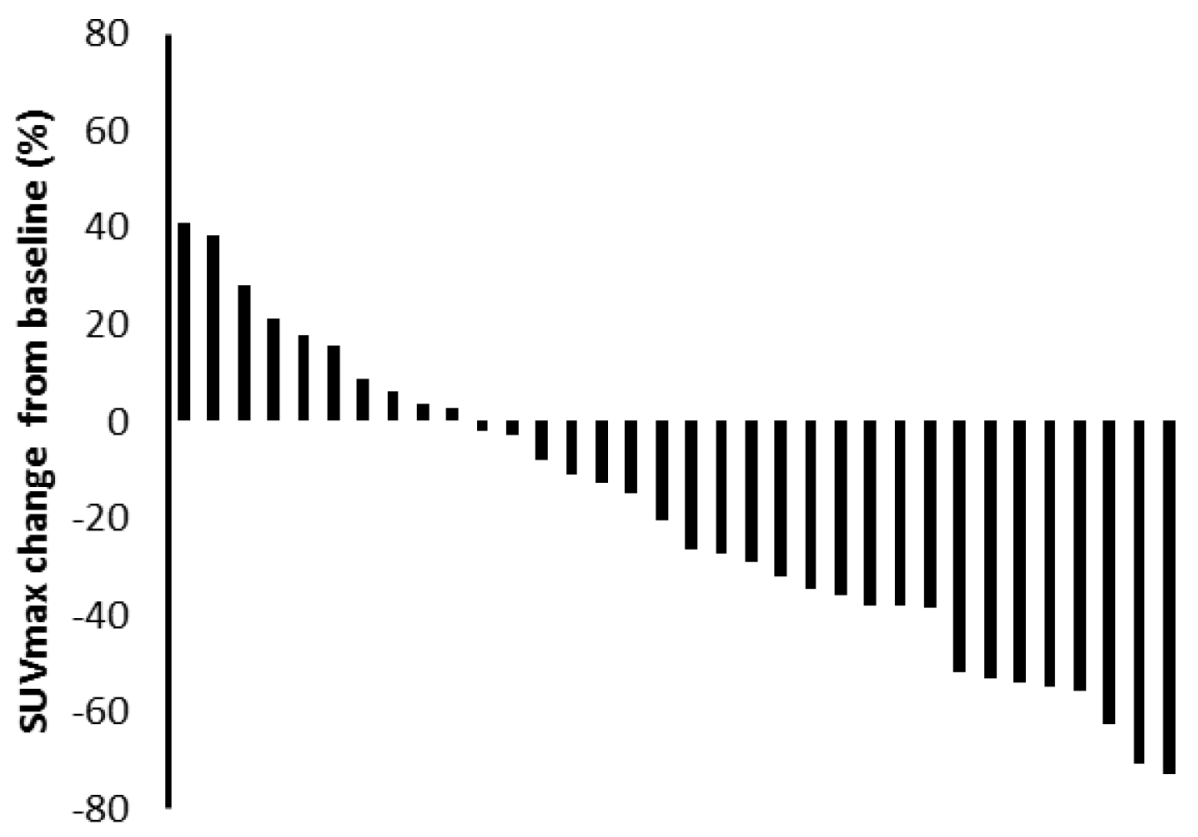

Lesions

Figure 1: A waterfall plot of posttreatment changes in SUVmax from baseline for thirty-four evaluable lesions.

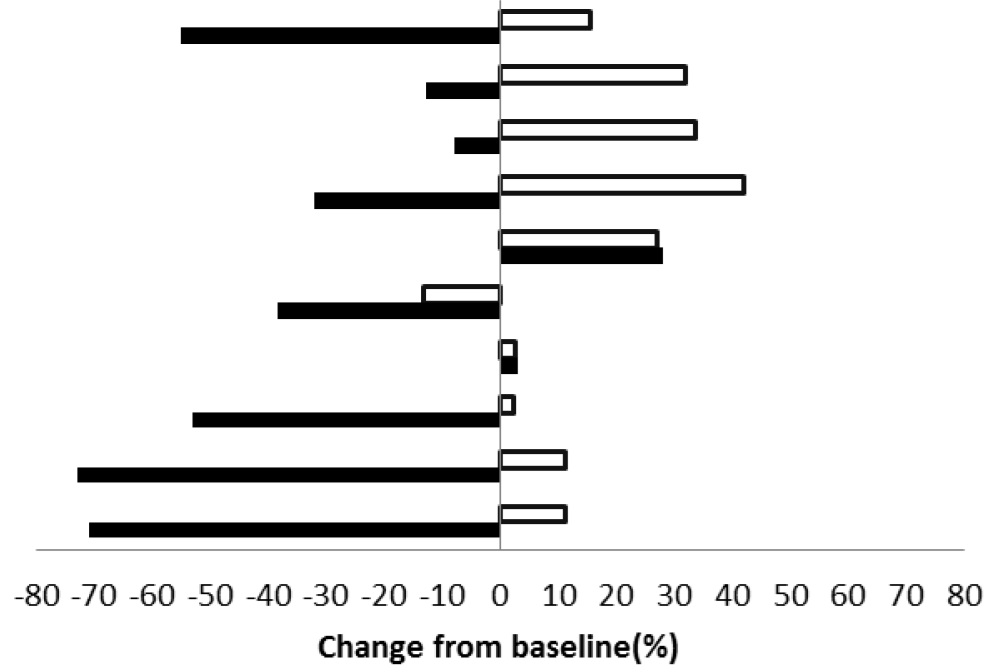

Figure 2: Maximum post treatment SUVmax versus tumor volume changes from baseline for each enrolled patient. (- indicates SUVmax change, $\square$ indicates tumor volume change).

donate the necessary fuels to anabolic cancer cells $[7,8]$. The non-proliferative and mitochondrial-poor stromal cells are probably dominant in the sarcoma tumor microenvironment, where CAFs usually have the largest increase in glucose uptake. [9] Thirdly, the MartinezOutschoorn's theory of "battery-operated tumor growth" might not be reversed by Rapa and HCQ treatment because the discrepancy of decreased glucose uptake with increased tumor volume may indicate that proliferative and mitochondrion-rich cancer cells still thrive after autophagy modulation therapy.
Rapa and newer generation mTOR inhibitors have limited clinical activity, except in certain tumor types with heavy dependence on mTOR [20,21]. Sarcoma is not one of them. Recently, either a Rapa analogue or HCQ has been used as an adjunctive anti-cancer agent in clinical trials [22-24]. The median decrease in SUVmax of $-23.7 \%$ by Rapa and HCQ treatment in our patient cohort cannot be considered an effective sarcoma treatment modality based on the current results. Since cancer cells use autophagy as a prosurvival mechanism during chemotherapy, we hypothesized that a strategy of combined Rapa, HCQ, and chemotherapy 

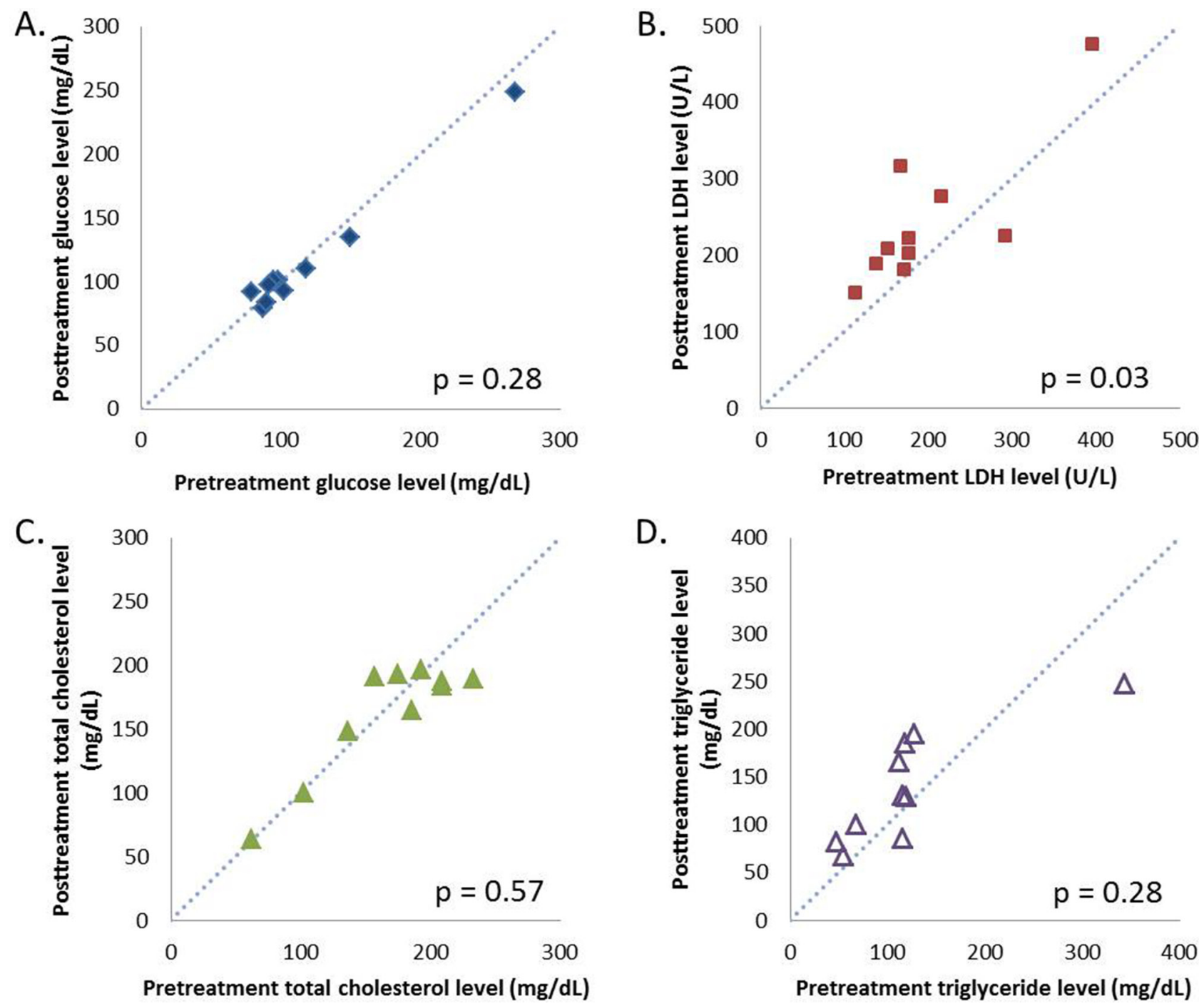

Figure 3: Calibration plots of fasting plasma glucose A. lactate dehydrogenase B. total cholesterol C. and triglyceride D. levels before and after two weeks of hydroxychloroquine and sirolimus treatment.

as a triplet combination should be more synergistic than only Rapa and HCQ doublet combination and might reverse drug resistance for the following reasons: (1.) The use of Rapa and chemotherapy might promote cancer cells (including sarcoma) to a higher autophagy state, while HCQ blocks their final autolysosome pathway and switches the pro-survival to pro-death process as an example of synthetic lethality [25], (2.) Systemic HCQ might block the vicious cycle of energy supply from the tumor microenvironment through the inhibition of CAF's autophagy, and (3.) The metabolic uncoupling of oxidative cancer cells from glycolytic stroma by Rapa and HCQ may render cancer cells more vulnerable to mitochondria-targeted drugs or oxidative stresses.

As shown in Figure 4, LDH levels significantly increased after treatment, which might indicate a compensatory process when aerobic glycolysis was inhibited by Rapa and HCQ. LDH levels might be used as surrogate biomarkers in future clinical trials. It has been reported that a dose-dependent rise in $\mathrm{LDH}$ after treatment with everolimus and octreotide correlated with better progressionfree survival on neuroendocrine tumors. [26] A rise in LDH in association with mTOR inhibition has been reported to be associated anti-angiogenesis associated hypoxia [27]. An everolimus and chloroquine combination has also been reported to inhibit angiogenesis [28]. Recent studies showed that the endothelial cells were one of the most critical tissues depending on aerobic glycolysis [29]. The rise in LDH shortly after treatment with autophagy modulators provide more evidence to suggest that a direct anti-glycolysis effect from the Rapa and HCQ doublet combination occurs in the tumor microenvironment.

\section{MATERIALS AND METHODS}

\section{Patients}

This study was reviewed and approved by our institutional review board. All patients provided written 


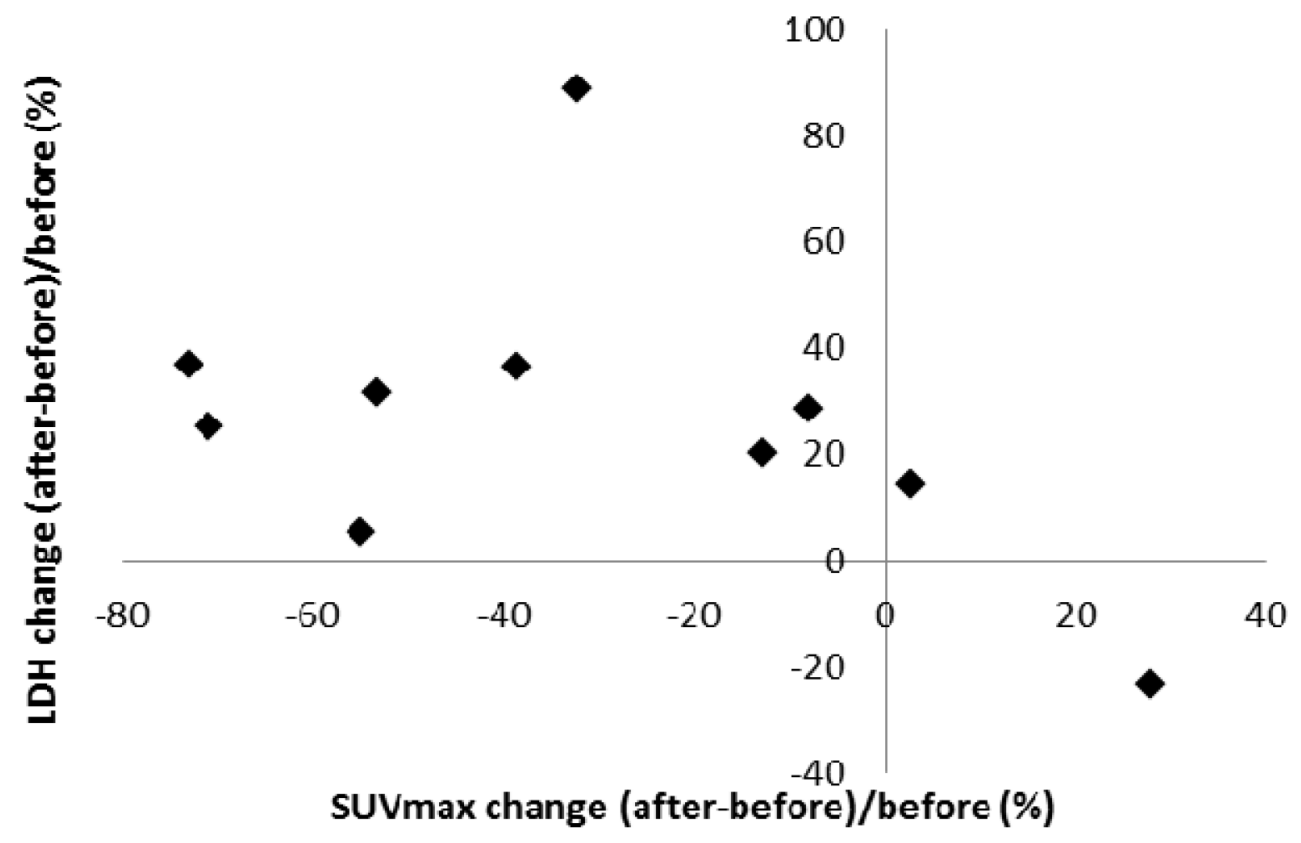

Figure 4: Correlation between SUVmax and lactate dehydrogenase level changes after two weeks of hydroxychloroquine and sirolimus treatment.

informed consent before participation. It was registered with ClinicalTrials.gov under registration No. NCT01842594. Patients with histologically confirmed sarcoma who failed first-line treatment were eligible. In addition, the eligibility criteria included at least one measurable lesion $(>2 \mathrm{~cm})$, an Eastern Cooperative Oncology Group performance status of $0-2$, as well as adequate hepatic, renal, and hematological functions (levels of serum bilirubin $\leq 25 \mathrm{mg} / \mathrm{dL}$, aspartate aminotransferase $\leq 5 \mathrm{x}$ institutional upper limit of normal, serum creatinine $\leq 2.0 \mathrm{mg} / \mathrm{dL}$, absolute neutrophil count $\geq 1.5 \times 10^{9} / \mathrm{L}$, and platelet count $\geq 100 \times 10^{9} / \mathrm{L}$ ). Patients were required to be free from any previous chemotherapy for more than five weeks before entering this study. Those with clinically significant cardiovascular diseases such as uncontrolled hypertension, myocardial infarction, unstable angina, or congestive heart failure of New York Heart Association grade II and higher were excluded.

\section{Procedures}

All of the patients underwent a physical examination and laboratory assessment of hematological, hepatic, and renal functions before entering the study. A baseline wholebody [18F]-FDG PET was performed before therapy initiation. Patients received $1 \mathrm{mg}$ of Rapa and $200 \mathrm{mg}$ of HCQ twice a day before meal. A second [18F]-FDG PET was performed two weeks later. Toxicity was assessed according to the National Cancer Institute Common Terminology Criteria version 3.0. No dose modification was allowed.

All of the PET scans were conducted using a Siemens Biograph mCt PET/CT scanner (Siemens Medical Solutions Inc., Molecular Imaging, IL, USA) with extended axial field of view (TrueV). The patients were required to fast for at least eight hours before the PET/CT scan. PET attenuation correction factors were calculated from CT images using low-dose CT (120 keV and $20 \mathrm{mAs}$ ) in shallow inspiration. The scanner had an average spatial resolution of $4.4 \mathrm{~mm}$ at $1 \mathrm{~cm}$ and $5.0 \mathrm{~mm}$ at $10 \mathrm{~cm}$ from the transverse field of view (FOV) and a maximum sensitivity of $8.1 \mathrm{kcps} /$ $\mathrm{MBq}$ at center of FOV. Its axial FOV was $21.6 \mathrm{~cm}$. After intravenous administration of $185 \mathrm{MBq}(5 \mathrm{mCi})$ of [18F]FDG, PET images were acquired for three minutes per bed position. The uptake period between the FDG injection and the beginning of PET scan was $60 \pm 10$ minutes (range, 50-70 minutes). The PET/CT scan started from the head and moved toward the pelvis; a head pillow and knee cushion were used to render positioning comparable in both scanners. Images were reconstructed via proprietary Siemens HD PET software using the iterative TrueX and time of flight ordered-subsets expectation maximization (TOF OSEM) method. They were displayed in three orthogonal projections and as a whole-body maximumpixel-intensity reprojection image for visual interpretation.

SUVs were calculated for all lesions. Regions of interest (ROI) were contoured to represent tumors $(>2 \mathrm{~cm})$ and organs (lungs, spleen, and liver) on all transaxial and coronal slices. ROIs were normalized for injection dose and body weight, and the maximum voxel value was recorded for each region or organ. The highest SUV measured with increased uptake was considered the SUVmax. Correlative diagnostic CT examinations were used for accurate localization. The most intense uptake at baseline was identified as the index lesion and evaluated for treatment response.

Two medical physicists independently analyzed the changes in SUVmax between baseline and post 


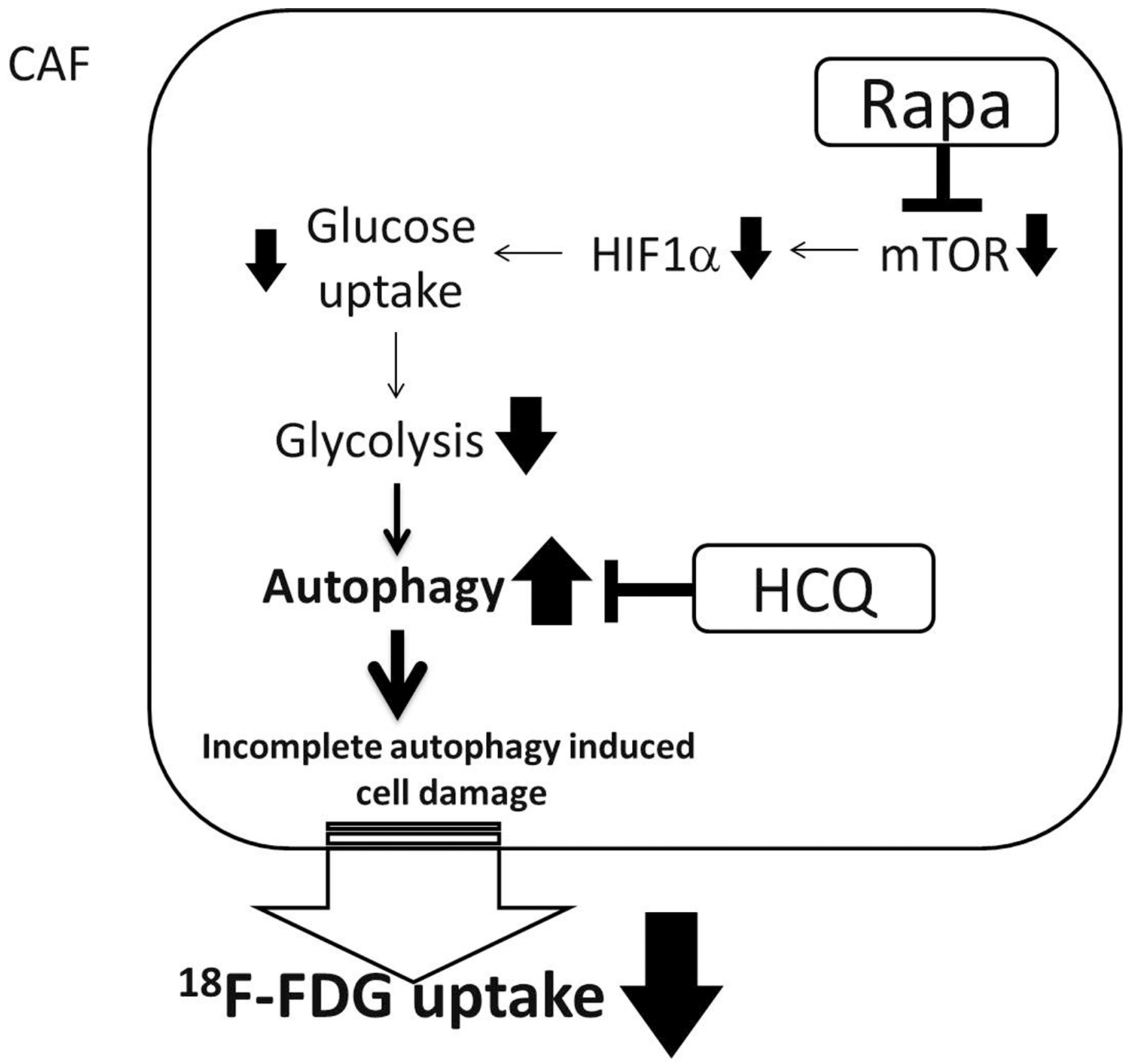

Figure 5: Synergistic effect of Rapa and HCQ combination. CAF cells are glycolytic-dependent cells. The combined use of Rapa and HCQ has synergistic effect on reducing glucose consumption rate.

treatment PET scans. Metabolic response after two weeks of treatment was evaluated according to the European Organization for Research and Treatment of Cancer (EORTC 1999) guidelines [12]. A complete response was defined as the complete resolution of $[18 \mathrm{~F}]-\mathrm{FDG}$ uptake within the tumor volume. A partial response was defined as a reduction of $25 \%$ or greater in tumor [18F]FDG uptake, whereas stable disease was defined as an increase or decrease in [18F]-FDG uptake of less than $25 \%$. Progressive disease was defined as an increase in [18F]-FDG uptake of $25 \%$ or higher, or as the appearance of a new [18F]-FDG uptake focus.

The CT images were forward to the Pinnacle treatment planning system, version 9.8 (Philips, Fitchburg,
WI, USA) where tumor sizes were measured on a digitalized image. An independent radiation oncologist ensured that the same lesions on SUVmax were measured and evaluated at pretreatment and on follow-up scans.

\section{Statistical analysis}

Percent changes from baseline SUVmax and tumor volume after Rapa and HCQ treatment were plotted as a waterfall chart. Differences in the results of comparative tests were determined by $t$-test and considered significant if the two-sided $p<0.05$. All statistical analysis was performed using the Statistical Application System software, SAS Version 9.1.3(SAS institute, Inc., Cary, NC, USA). 


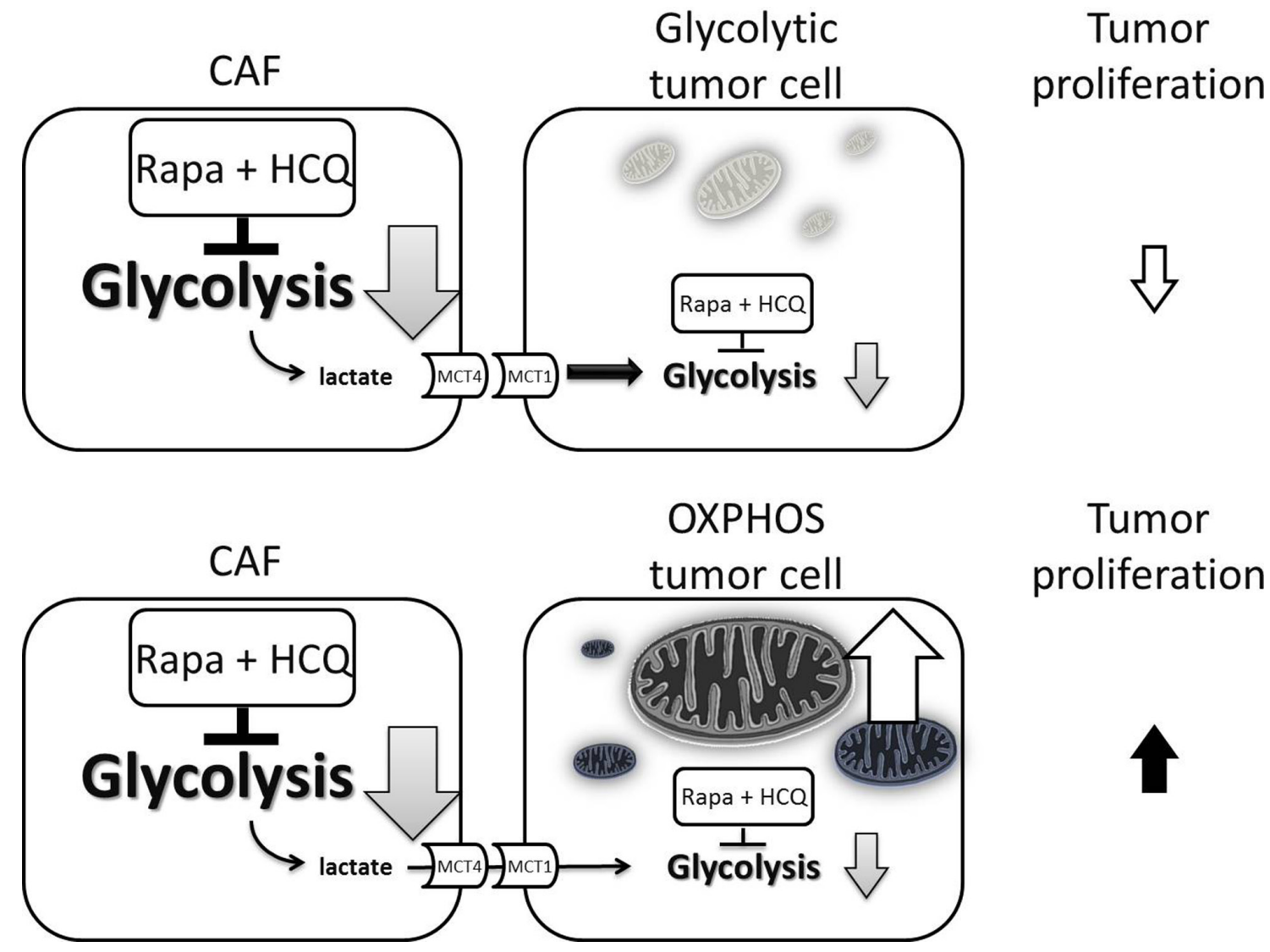

Figure 6: Model of uncoupling energy transfer within sarcoma tumor. Rapa and HCQ combination will inhibit the aerobic glycolysis and reduce the energy transfer from CAFs to OXPHOS sarcoma cells and glycolytic sarcoma cells. The decreased lactate supply has little effect on OXPHOS sarcoma cells due to their independent energy sources, their mitochondria.

\section{CONCLUSIONS}

The cancer metabolism pathway compartmentalization model (oxidative vs glycolytic) is still in its infancy due to limited clinical methods for direct study. The combination treatment with Rapa and HCQ in sarcoma patients by [18f]-FDG-PET revealed a reduction of 2-deoxyglucose uptake while increasing tumor volume, which fits the catabolic tumor fibroblasts and anabolic cancer cells model well. The reversal of "reversed Warburg effect" in CAFs by the combination of autophagy inducers (Rapa) and autophagy inhibitor (HCQ) may offer opportunities for treatment strategies targeted on the deregulated metabolism in tumor microenvironment.

\section{REFERENCES}

1. Farhidzadeh H, Zhou M, Goldgof DB, Hall LO, Raghavan M, Gatenby RA. Prediction of treatment response and metastatic disease in soft tissue sarcoma. SPIE 9035, Medical Imaging 2014: Computer-Aided Diagnosis, 903518; doi: $10.1117 / 12.2043792$.
2. Klemm F, Joyce JA. Microenvironmental regulation of therapeutic response in cancer. Trends Cell Biol. 2015; 25:198-213.

3. Valcz G, Sipos F, Tulassay Z, Molnar B, Yagi Y. Importance of carcinoma-associated fibroblast-derived proteins in clinical oncology. J Clin Pathol. 2014; 67:1026-1031.

4. Madar S, Goldstein I, Rotter V. 'Cancer associated fibroblasts' - more than meets the eye. Trends Mol Med. 2013; 19:447-453.

5. Martinez-Outschoorn UE, Whitaker-Menezes D, Pavlides S, Chiavarina B, Bonuccelli G, Casey T, Tsirigos A, Migneco G, Witkiewicz A, Balliet R, Mercier I, Wang C, Flomenberg N, Howell A, Lin Z, Caro J, et al. The autophagic tumor stroma model of cancer or "battery-operated tumor growth": A simple solution to the autophagy paradox. Cell Cycle. 2010; 9:4297-4306.

6. Martinez-Outschoorn UE, Pavlides S, Howell A, Pestell RG, Tanowitz HB, Sotgia F, Lisanti MP. Stromal-epithelial metabolic coupling in cancer: integrating autophagy and metabolism in the tumor microenvironment. Int $\mathrm{J}$ Biochem Cell Biol. 2011; 43:1045-51. 
7. Lisanti MP, Sotgia F, Pestell RG, Howell A, MartinezOutschoorn UE. Stromal glycolysis and MCT4 are hallmarks of DCIS progression to invasive breast cancer. Cell Cycle. 2013; 12:2935-2936.

8. Lisanti MP, Martinez-Outschoorn UE, Sotgia F. Oncogenes induce the cancer-associated fibroblast phenotype: metabolic symbiosis and "fibroblast addiction" are new therapeutic targets for drug discovery. Cell Cycle. 2013; 12:2723-2732.

9. Kinnaird A, Michelakis ED. Metabolic modulation of cancer: a new frontier with great translational potential. J Mol Med (Berl). 2015; 93:127-42.

10. Martinez-Outschoorn U, Sotgia F, Lisanti MP. Tumor microenvironment and metabolic synergy in breast cancers: critical importance of mitochondrial fuels and function. Semin Oncol. 2014; 41:195-216.

11. Martinez-Outschoorn UE, Lin Z, Trimmer C, Flomenberg N, Wang C, Pavlides S, Pestell RG, Howell A, Sotgia F, Lisanti MP. Cancer cells metabolically "fertilize" the tumor microenvironment with hydrogen peroxide, driving the Warburg effect: Implications for PET imaging of human tumors. Cell Cycle. 2011; 10:2504-2520.

12. Young H, Baum R, Cremerius U, Herholz K, Hoekstra O, Lammertsma AA, Pruim J, Price P. Measurement of clinical and subclinical tumour response using [18F]-fluorodeoxyglucose and positron emission tomography: review and EORTC recommendations. European Organization for Research and Treatment of Cancer (EORTC) PET Study Group. Eur J Cancer. 1999; 35:1773-1782.

13. Martinez-Outschoorn UE, Balliet RM, Rivadeneira DB, Chiavarina B, Pavlides S, Wang C, Whitaker-Menezes D, Daumer KM, Lin Z, Witkiewicz AK, Flomenberg N, Howell A, Pestell RG, Knudsen ES, Sotgia F, Lisanti MP. Oxidative stress in cancer associated fibroblasts drives tumor-stroma co-evolution: A new paradigm for understanding tumor metabolism, the field effect and genomic instability in cancer cells. Cell Cycle. 2010; 9:3256-3276.

14. Martinez-Outschoorn UE, Trimmer C, Lin Z, WhitakerMenezes D, Chiavarina B, Zhou J, Wang C, Pavlides S, Martinez-Cantarin MP, Capozza F, Witkiewicz AK, Flomenberg N, Howell A, Pestell RG, Caro J, Lisanti MP, et al. Autophagy in cancer associated fibroblasts promotes tumor cell survival: Role of hypoxia, HIF1 induction and NFKB activation in the tumor stromal microenvironment. Cell Cycle. 2010; 9:3515-3533.

15. Martinez-Outschoorn UE, Pavlides S, Whitaker-Menezes D, Daumer KM, Milliman JN, Chiavarina B, Migneco G, Witkiewicz AK, Martinez-Cantarin MP, Flomenberg N, Howell A, Pestell RG, Lisanti MP, Sotgia F. Tumor cells induce the cancer associated fibroblast phenotype via caveolin-1 degradation: implications for breast cancer and DCIS therapy with autophagy inhibitors. Cell Cycle. 2010; 9:2423-2433.
16. Martinez-Outschoorn UE, Lisanti MP, Sotgia F. Catabolic cancer-associated fibroblasts transfer energy and biomass to anabolic cancer cells, fueling tumor growth. Semin Cancer Biol. 2014; 25:47-60.

17. Sotgia F, Martinez-Outschoorn UE, Lisanti MP. The reverse Warburg effect in osteosarcoma. Oncotarget. 2014; 5:7982-7983.

18. Bonuccelli G, Avnet S, Grisendi G, Salerno M, Granchi D, Dominici M, Kusuzaki K, Baldini N. Role of mesenchymal stem cells in osteosarcoma and metabolic reprogramming of tumor cells. Oncotarget. 2014; 5:7575-7588.

19. Nogova L, Boellaard R, Kobe C, Hoetjes N, Zander T, Gross SH, Dimitrijevic S, Pellas T, Eschner W, Schmidt K, Bangard C, Hayes W, Thomas RK, Dietlein M, Giaccone G, Hoekstra OS, et al. Downregulation of 18F-FDG uptake in PET as an early pharmacodynamic effect in treatment of non-small cell lung cancer with the mTOR inhibitor everolimus. J Nucl Med. 2009; 50:1815-1819.

20. Motzer RJ, Escudier B, Oudard S, Hutson TE, Porta C, Bracarda S, Grunwald V, Thompson JA, Figlin RA, Hollaender N, Urbanowitz G, Berg WJ, Kay A, Lebwohl D, Ravaud A. Efficacy of everolimus in advanced renal cell carcinoma: a double-blind, randomised, placebo-controlled phase III trial. Lancet. 2008; 372:449-456.

21. Hasskarl J. Everolimus. Recent Results Cancer Res. 2014; 201:373-392.

22. Andre F, O'Regan R, Ozguroglu M, Toi M, Xu B, Jerusalem G, Masuda N, Wilks S, Arena F, Isaacs C, Yap YS, Papai Z, Lang I, Armstrong A, Lerzo G, White M, et al. Everolimus for women with trastuzumab-resistant, HER2positive, advanced breast cancer (BOLERO-3): a randomised, double-blind, placebo-controlled phase 3 trial. Lancet Oncol. 2014; 15:580-591.

23. Sotelo J, Briceno E, Lopez-Gonzalez MA. Adding chloroquine to conventional treatment for glioblastoma multiforme: a randomized, double-blind, placebo-controlled trial. Ann Intern Med. 2006; 144:337-343.

24. Rangwala R, Chang YC, Hu J, Algazy KM, Evans TL, Fecher LA, Schuchter LM, Torigian DA, Panosian JT, Troxel AB, Tan KS, Heitjan DF, DeMichele AM, Vaughn DJ, Redlinger M, Alavi A, et al. Combined MTOR and autophagy inhibition: phase I trial of hydroxychloroquine and temsirolimus in patients with advanced solid tumors and melanoma. Autophagy. 2014; 10:1391-1402.

25. Wang Y-S, Huang Y-C, Fong W-L, Chi M-S, Chi K-H. Abstract 5611: A synthetic lethality combination with chloroquine and rapamycin on chemosensitization Proceedings: AACR 103rd Annual Meeting 2012; Chicago, IL.

26. Yao JC, Phan AT, Chang DZ, Wolff RA, Hess K, Gupta S, Jacobs C, Mares JE, Landgraf AN, Rashid A, Meric-Bernstam F. Efficacy of RAD001 (everolimus) and octreotide LAR in advanced low- to intermediate-grade neuroendocrine tumors: results of a phase II study. J Clin Oncol. 2008; 26:4311-4318. 
27. Lukacova S, Sorensen BS, Alsner J, Overgaard J, Horsman MR. The impact of hypoxia on the activity of lactate dehydrogenase in two different pre-clinical tumour models. Acta Oncol. 2008; 47:941-947.

28. Grimaldi A, Balestrieri ML, D’Onofrio N, Di Domenico G, Nocera C, Lamberti M, Tonini G, Zoccoli A, Santini D, Caraglia M, Pantano F. The Synergistic Effect of Everolimus and Chloroquine on Endothelial Cell Number Reduction Is Paralleled by Increased Apoptosis and Reduced Autophagy Occurrence. PLoS ONE. 2013; 8:e79658.

29. Stapor P, Wang X, Goveia J, Moens S, Carmeliet P. Angiogenesis revisited - role and therapeutic potential of targeting endothelial metabolism. J Cell Sci. 2014; 127:4331-41. 